\title{
Asymptotic behavior of rarefaction waves for a model system of a radiating gas
}

Qinghua Xiao*, Yuning Liu and Jongsung Kim

* Correspondence:

2007xiaoqinghua@163.com

School of Mathematics and

Statistics, Wuhan University, Wuhan

430072, China

\begin{abstract}
This article is concerned with nonlinear stability together with the corresponding convergence rates of rarefaction waves for a hyperbolic-elliptic coupled system arising in the 1D motion of a radiating gas with large initial perturbation. Compared with former results, although we ask the $L^{2}(\mathbf{R})$-norm of the initial perturbation to be small but the $L^{2}(\mathbf{R})$-norm of the first- and second-order derivatives of the initial perturbation with respect to the spatial variable $x$ can be large and consequently, the $H^{2}(\mathbf{R})$-norm of the initial perturbation can be large.
\end{abstract}

AMS Subject Classifications: 34K25; 35M10.

Keywords: hyperbolic-elliptic coupled system, rarefaction waves, convergence rates, large initial perturbation, continuation argument

\section{Introduction}

This article is concerned with the large time asymptotic behavior of the global solution to the Cauchy problem for the following hyperbolic-elliptic coupled system

$$
\left\{\begin{array}{l}
u_{t}+f(u)_{x}+q_{x}=0 \\
-q_{x x}+q+u_{x}=0
\end{array}\right.
$$

with initial data

$$
\left.u(t, x)\right|_{t=0}=u_{0}(x) \rightarrow u_{ \pm}, \quad x \rightarrow \pm \infty .
$$

Here $f(u)$ is a sufficiently smooth function satisfying

$$
f^{\prime \prime}(u) \geq a>0
$$

for some positive constant $a$ and all $u \in \mathbf{R}$ under consideration and $u_{-}<u_{+}$are two given constants.

When $f(u)=\frac{1}{2} u^{2}$, the system (1.1) is a simple model for the $1 \mathrm{D}$ motion of a radiating gas, cf. [1]. In fact, $u$ and $q$ in (1.1) represent the velocity and the heat flux of the gas, respectively.

As $t \rightarrow \infty$, the asymptotic behavior of the global solutions of the Cauchy problem (1.1), (1.2) is closely related to that of the Riemann problem for the corresponding scalar conservation law 


$$
\left\{\begin{array}{l}
w_{t}^{R}+f\left(w^{R}\right)_{x}=0 \\
\left.w^{R}(t, x)\right|_{t=0}=w_{0}^{R}(x)=\left\{\begin{array}{l}
u_{-}, x<0 \\
u_{+}, x>0
\end{array}\right.
\end{array}\right.
$$

Under the assumption (1.3), the fact $u_{-}<u_{+}$implies that the Riemann problem (1.4) admits a unique global entropy (rarefaction wave) solution $w^{R}(t, x)=w^{R}\left(\frac{x}{t}\right)$ defined by

$$
w^{R}\left(\frac{x}{t}\right)= \begin{cases}u_{-}, & x \leq f^{\prime}\left(u_{-}\right) t \\ \left(f^{\prime}\right)^{-1}\left(\frac{x}{t}\right), & f^{\prime}\left(u_{-}\right) t \leq x \leq f^{\prime}\left(u_{+}\right) t \\ u_{+}, & x \geq f^{\prime}\left(u_{+}\right) t .\end{cases}
$$

The main purpose of our present article is to study the nonlinear stability of such a $w^{R}(t, x)$. To do so, as in [2], one needs first to introduce a smooth approximation $w(t$, $x)$ of the rarefaction wave solution $w^{R}(t, x)$. Since such a $w(t, x)$ tends to $w^{R}(t, x)$ as $t$ $\rightarrow \infty$ (cf. Lemma 2.1 below), thus the study of the above problem is then transferred to show that if the initial data $u_{0}(x)$ is a perturbation of $w(0, x)$, the Cauchy problem (1.1), (1.2) admits a unique global solution $(u(t, x), q(t, x))$ which tends to $\left(w(t, x),-\partial_{x} w\right.$ $(t, x))$ as $t \rightarrow \infty$. Recall that depending on whether $\delta=u_{+}-u_{-}$, the strength of the rarefaction waves, and/or certain Sobolev norm, cf. $\left\|u_{0}(x)-w_{0}^{R}(x)\right\|_{L^{2}(\mathbf{R})}+\left\|\partial_{x} u_{0}(x)\right\|_{H^{1}(\mathbf{R})}$, of the initial perturbation $u_{0}(x)-w(0, x)$ are assumed to be small or not, the corresponding stability results are called local (or global) nonlinear stability of strong (or weak) rarefaction waves, respectively.

For such a problem, when $f(u)=\frac{1}{2} u^{2}$, by introducing the smooth approximation $\tilde{w}(t, x)$ of the rarefaction wave solution $w^{R}(t, x)$ as the unique solution of the following Cauchy problem

$$
\left\{\begin{array}{l}
\tilde{w}_{t}+\tilde{w} \tilde{w}_{x}=\tilde{w}_{x x}, \quad t>-t_{0}, x \in \mathbf{R} \\
\tilde{w}\left(-t_{0}, x\right)=w_{0}^{R}(x), x \in \mathbf{R}
\end{array}\right.
$$

and based on some delicate estimates on such a $\tilde{w}(t, x)$ which are obtained by exploiting the celebrated Hopf-Cole transformation, the authors showed in [1] that rarefaction waves of the Cauchy problem (1.1), (1.2) is indeed nonlinear stable provided that $\delta$ and the $H^{2}(\mathbf{R})$-norm of the initial perturbation, i.e., $\left\|u_{0}(x)-\tilde{w}(0, x)\right\|_{H^{2}(\mathbf{R})}$ are sufficiently small. Moreover, it is shown in [1] that for $t>0$ sufficiently large, the following temporal decay estimates

$$
\left\{\begin{array}{l}
\left\|u(t, x)-w^{R}\left(\frac{x}{t}\right)\right\|_{L^{p}(\mathbf{R})} \leq O(1)(1+t)^{-\frac{1}{2}\left(1-\frac{1}{p}\right)}, \quad 1<p \leq \infty, \\
\left\|q(t, x)+\partial_{x} w^{R}\left(\frac{x}{t}\right)\right\|_{L^{p}(\mathbf{R})} \leq O(1)(1+t)^{-\frac{1}{2}\left(2-\frac{1}{p}\right)}, \frac{4}{3} \leq p \leq \infty
\end{array}\right.
$$

hold if one assumes further that $u_{0}(x)-\tilde{w}(0, x) \in L^{1}(\mathbf{R})$.

Since the results obtained in [1] only implies that weak rarefaction waves of the Cauchy problem (1.1), (1.2) is locally nonlinear stable, thus a question of some interesting 
is: Can the assumption that $\left\|u_{0}(x)-\tilde{w}(0, x)\right\|_{H^{2}(\mathbf{R})}$ is sufficiently small be weakened or even be removed such that rarefaction waves of the Cauchy problem (1.1), (1.2) are global nonlinear stable? Moreover, if the rarefaction waves are nonlinear stable for such a class of large initial perturbation, does the same convergence rate as in [1] hold for such a class of perturbation?

This article is concentrated on these problems and our main result can be stated as follows.

Theorem 1.1 (Nonlinear stability and convergence rates) Let $u_{0}(x)-w_{0}^{R}(x) \in L^{1}(\mathbf{R}) \cap L^{2}(\mathbf{R}), \delta_{x} u_{0}(x) \in H^{1}(\mathbf{R})$ and suppose further that there exist positive constants $D_{1}, D_{2}, D_{3}, \beta$, and $\gamma$, which are independent of $\delta>0$, such that

$$
\left\{\begin{aligned}
\left\|u_{0}(x)-w_{0}^{R}(x)\right\|_{L^{2}(\mathbf{R})}^{2} & \leq D_{1} \delta^{\beta} \\
\left\|\partial_{x} u_{0}(x)\right\|_{L^{2}(\mathbf{R})}^{2} & \leq D_{2}\left(1+\delta^{-\gamma}\right) \\
\left\|\partial_{x}^{2} u_{0}(x)\right\|_{L^{2}(\mathbf{R})}^{2} & \leq D_{3}\left(1+\delta^{-\gamma}\right)
\end{aligned}\right.
$$

then if

$$
2 \geq \beta>3 \gamma,
$$

there is a sufficiently small constant $\delta_{0}>0$ such that when $0<\delta \leq \delta_{0}$, the Cauchy problem (1.1), (1.2) admits a unique global solution $(u(t, x), q(t, x))$ satisfying

$$
\begin{aligned}
& u(t, x)-w(t, x) \in L^{\infty}\left([0, \infty) ; H^{2}(\mathbf{R})\right) \cap C^{0}\left([0, \infty) ; H^{1}(\mathbf{R})\right), \\
& q(t, x)+w_{x}(t, x) \in L^{\infty}\left([0, \infty) ; H^{3}(\mathbf{R})\right) \cap C^{0}\left([0, \infty) ; H^{2}(\mathbf{R})\right) .
\end{aligned}
$$

Here $w(t, x)=\left(f^{\prime}\right)^{-1}(\tilde{w}(t, x))$.

Moreover, for $t>0$ sufficiently large, the solution $(u(t, x), q(t, x))$ verifies

$$
\left\|u(t, x)-w^{R}\left(\frac{x}{t}\right)\right\|_{L^{p}(\mathbf{R})} \leq O(1)(1+t)^{-\frac{1}{2}\left(1-\frac{1}{p}\right)}(\ln (2+t))^{\frac{7 p-4}{3 p}}, \quad 1 \leq p \leq+\infty .
$$

and

$$
\left\|q(t, x)-\partial_{x} w^{R}\left(\frac{x}{t}\right)\right\|_{L^{p}(\mathrm{R})} \leq O(1)(1+t)^{-\frac{1}{2}\left(2-\frac{1}{p}\right)}(\ln (2+t))^{\frac{21 p-8}{5 p}}, \quad \frac{4}{3} \leq p \leq+\infty .
$$

Here $O(1)$ denotes some positive constant which depends on $\left\|u_{0}(x)-w(0, x)\right\|_{H^{1}(\mathbf{R}) \cap L^{1}(\mathbf{R})}$.

Remark 1.1 Since $\delta$ in Theorem 1.1 is assumed to be sufficiently small, the assumption (1.8) does imply that the $L^{2}(\mathbf{R})$-norm of the initial perturbation is small, but the $L^{2}$ (R)-norm of the first and second derivatives of the initial perturbation with respect to $x$ can be indeed large and consequently, compared with that of [1], the $H^{2}(\mathbf{R})$-norm of the initial perturbation can indeed be large. We note, however, that although we obtain some result on the nonlinear stability of rarefaction waves of the Cauchy problem (1.1), (1.2) for a class of initial perturbation which is large in $H^{2}(\mathbf{R})$, the problem on the nonlinear stability of strong rarefaction waves of the Cauchy problem (1.1), (1.2) for any 
initial perturbation in $H^{2}(\mathbf{R})$ is still not known and will be pursued by the authors in the future.

Remark 1.2 Notice that the decay estimates (1.12) and (1.13) are weaker than that of (1.7), but it is worth to pointing out that when $f(u)=\frac{1}{2} u^{2}$, we can also show that (1.7) holds only under the conditions listed in Theorem 1.1. It would be of some interest to show whether (1.7) holds for general smooth nonlinear flux function $f(u)$.

Now we outline the main ingredients used in deducing our main results. To prove Theorem 1.1, as in [1], we only need to deduce certain estimates on $(\varphi(t, x), \psi(t, x))=$ $\left(u(t, x)-w(t, \quad x), q(t, \quad x)+\partial_{x} w(t, \quad x)\right)$ since the estimates on $\left(w(t, x)-w^{R}\left(\frac{x}{t}\right), \partial_{x} w(t, x)-\partial_{x} w^{R}\left(\frac{x}{t}\right)\right)$ are well-established in [1].

Our analysis is based on an elementary energy method together with the continuation argument and the key point is how to control the possible growth of the solution caused by the nonlinearity of the equation under consideration. To state our argument clearly, let's recall that the analysis in [1] is first to perform some energy estimates based on the a priori assumption that

$$
N(t):=\sup _{0 \leq \tau \leq t}\|u(\tau, x)-w(\tau, x)\|_{H^{2}(\mathbf{R})} \leq \eta
$$

holds for some positive constant $\eta>0$ which is chosen sufficiently small, and then to use the continuation argument to extend the local solution step by step to a global one. To do so, the argument in [1] is to use the smallness of $\eta$ and $\delta$ to control the possible growth of the solution caused by the nonlinearity of the equation and consequently this argument can only lead to the local stability of weak rarefaction waves. Our main idea, however, is to perform the same energy estimates based on the a priori assumption that

$$
N(t):=\sum_{i=0}^{1} \sup _{0 \leq \tau \leq t}\left\|\frac{\partial^{i}(u(\tau, x)-w(\tau, x))}{\partial x^{i}}\right\|_{L^{\infty}(\mathbf{R})} \leq \eta
$$

for some $\eta>0$ and our main trick is to use the smallness of $\eta$ and $\delta$ to control the possible growth of the solution caused by the nonlinearity of the equation. Thus to use the continuation argument to extend the local solution step by step to a global one, a key step is how to design a class of initial perturbation, which is large in the $H^{2}$-norm, so that the scheme can be continued. It is worth to pointing out in this step that we ask the initial perturbation to satisfy (1.8).

Another purpose of this article is to show that if the Cauchy problem (2.12)-(2.14) admits a unique global solution $(\varphi(t, x), \psi(t, x))=\left(u(t, x)-w(t, x), q(t, x)+\partial_{x} w(t, x)\right)$ and there exists some positive constant $E$, which is independent of $t, x$, and $\delta$ for sufficiently small $\delta>0$, such that

$$
\|\phi(t)\|_{L^{\infty}(\mathbf{R})}+\left\|\phi_{x}(t)\right\|_{L^{\infty}(\mathbf{R})} \leq E, \quad \forall t \geq 0
$$

and the rarefaction wave is nonlinear stable, i.e.,

$$
\lim _{t \rightarrow \infty}\left(\|\phi(t)\|_{L^{\infty}(\mathbf{R})}+\left\|\phi_{x}(t)\right\|_{L^{\infty}(\mathbf{R})}\right)=0,
$$


then we can indeed show that (1.12) and (1.13) holds provided that $u_{0}(x)-w(0, x) \in$ $H^{1}(\mathbf{R}) \cap L^{1}(\mathbf{R})$. Such a result implies that if one can show that the Cauchy problem (2.12)-(2.14) admits a unique global solution $(\varphi(t, x), \psi(t, x))$ which satisfies (1.16) and (1.17), then even for any initial perturbation and any $\delta>0$, one can deduce (1.12) and (1.13) provided that $u_{0}(x)-w(0, x) \in H^{1}(\mathbf{R}) \cap L^{1}(\mathbf{R})$.

Before concluding this section, we point out that the study on the nonlinear stability of rarefaction waves to hyperbolic conservation laws with dissipative terms was initiated by Il'in and Oleinik [3] and since then many excellent results have been obtained, the interested author is referred to [1-17] and the references cited therein.

This article is arranged as follows: reformulation of the problem and some estimates on $w^{R}(t, x)$ together with its smooth approximation will be stated in Section 2, nonlinear stability of the rarefaction waves for a class of large initial data will be discussed in Sections 3 and 4 is devoted to deducing some decay estimate.

Notations: Throughout the rest of this article, $C$ or $O(1)$ will be used to denote a generic positive constants which may vary from line to line. Let's emphasize that unless stated clearly, all the constants are independent of $t, x$, and $\delta$ for sufficiently small $\delta>0$.

$H^{l}(\mathbf{R})(l \geq 0)$ and $L^{p}(\mathbf{R})$ stand for the usual Sobolev space with the norm $\|\cdot\|_{H^{l}(\mathbf{R})}$ and the usual Lebesgue space with the norm $\|\cdot\|_{L^{p}(\mathbf{R})}$, respectively. While $\|\cdot\|_{L^{p}}(1 \leq p \leq+\infty),\|\cdot\|_{l}$ will be used to denote the usual $L^{p}(\mathbf{R})$-norm, $H^{l}(\mathbf{R})$-norm and $f^{(j)}(u)=\frac{d^{j} f(u)}{d u^{j}}$ for $j \in \mathbf{N}$. For simplicity, $\|f(t, \cdot)\|_{L^{p}(\mathbf{R})}$ and $\|f(t, \cdot)\|_{H^{l}(\mathbf{R})}$ will usually be denoted by $\|f(t)\|_{L^{p}}$ and $\|f(t)\|_{l}$, respectively. Especially, $\|\cdot\|_{L^{2}(\Omega)}=\|\cdot\|$.

\section{Reformulation of the problem}

First, we introduce the smooth approximation of the rarefaction wave $w^{R}(t, x)$. For this purpose, let $w(t, x)=\left(f^{\prime}\right)^{-1}(\tilde{w}(t, x))$, where $\tilde{w}(t, x)$, is the solution of $(1.6)_{1}$ with $\tilde{w}\left(-t_{0}, x\right)=f^{\prime}\left(w_{0}^{R}(x)\right)$, then $w(t, x)$ is the solution of the Cauchy problem

$$
\left\{\begin{array}{l}
w_{t}+f(w)_{x}=w_{x x}+\frac{f^{\prime \prime \prime}(w)}{f^{\prime \prime}(w)} w_{x^{\prime}}^{2} \\
\left.w(t, x)\right|_{t=0}=w_{0}(x)=\left(f^{\prime}\right)^{-1}(\tilde{w}(0, x)) .
\end{array}\right.
$$

Now we collect some properties of $w^{R}(t, x)$ and $w(t, x)$ given in [1].

Lemma 2.1 For $1 \leq p \leq \infty$ and $t>0$, there exist positive constants $C$ and $C_{p}$ which are independent of $x, t$, and $\delta$ for $\delta>0$ sufficiently small such that

$$
\begin{aligned}
& \left\|w^{R}\left(t+t_{0}, \cdot\right)-w^{R}(t, \cdot)\right\|_{L^{p}} \leq \min \left\{C \delta, C \delta \bar{p}_{t}^{-\left(1-\frac{1}{p}\right)}\right\}, \\
& \left\|\partial_{x} w^{R}\left(t+t_{0}, \cdot\right)-\partial_{x} w^{R}(t, \cdot)\right\|_{L^{p}} \leq \min \left\{C \delta \bar{p}_{t}^{-\left(1-\frac{1}{p}\right)}, C t^{-1}\right\}, \\
& w_{x}(t, x)>0,
\end{aligned}
$$




$$
\begin{aligned}
& \left\|w^{R}\left(t_{0}, \cdot\right)-w(0, \cdot)\right\|_{L^{p}} \leq C \delta, \\
& \left\|w^{R}\left(t+t_{0}, \cdot\right)-w(t, \cdot)\right\|_{L^{p}} \leq C(1+t)^{-\frac{1}{2}\left(1-\frac{1}{p}\right)}, \\
& \left\|\partial_{x} w^{R}\left(t+t_{0}, \cdot\right)-\partial_{x} w(t, \cdot)\right\|_{L^{p}} \leq C(1+t)^{-\left(1-\frac{1}{p}\right)}, \\
& \left\|\partial_{x} w(t, \cdot)\right\|_{L^{p}} \leq C_{p} \delta^{\frac{1}{p}}(1+t) \\
& \left\|\partial_{x}^{k} w(t, \cdot)\right\|_{L^{p}} \leq C \delta(1+t)^{\left.-\frac{1}{p}\right)}, \\
& \left\|\partial_{x}^{k} w(t, \cdot)\right\|_{L^{p}} \leq C(1+t)^{\left.-\frac{1}{2}\right)}, k \geq 1, \\
& \|^{\left(k+1-\frac{1}{p}\right)}, k \geq 2 .
\end{aligned}
$$

Denote the perturbation $(\varphi(t, x), \psi(t, x))$ as follows

$$
u=w+\phi, \quad q=-w_{x}+\psi,
$$

then (1.1), (1.2) can be rewritten as

$$
\begin{aligned}
& \phi_{t}+(f(w+\phi)-f(w))_{x}+\psi_{x}=-\frac{f^{\prime \prime \prime}(w)}{f^{\prime \prime}(w)} w_{x}^{2} \\
& -\psi_{x x}+\psi+\phi_{x}=-w_{x x x}
\end{aligned}
$$

and

$$
\phi(0, x)=\phi_{0}(x)=u_{0}(x)-w_{0}(x) .
$$

For the reformulated problem (2.12)-(2.14), we can deduce the following result.

Theorem 2.1 (Nonlinear stability result) Suppose that $\varphi_{0}(x) \in H^{2}(\mathbf{R})$ and verifies the conditions

$$
\begin{aligned}
& \left\|\phi_{0}(x)\right\|^{2} \leq D_{1} \delta^{\beta}, \\
& \left\|\phi_{0}^{\prime}(x)\right\|^{2} \leq D_{2}\left(1+\delta^{-\gamma}\right), \\
& \left\|\phi_{0}^{\prime \prime}(x)\right\|^{2} \leq D_{3}\left(1+\delta^{-\gamma}\right) .
\end{aligned}
$$

Here $D_{i}(i=1,2,3)$ are positive constants independent of the positive constant $\delta$ and the constants $\beta, \gamma$ satisfying (1.9).

Then there is a sufficiently small constant $\delta_{0}>0$ such that if $0<\delta<\delta_{0}$, the problem (2.12)-(2.14) admits a unique global solution $(\varphi(t, x), \psi(t, x))$ satisfying 


$$
\left\{\begin{array}{l}
\phi(t, x) \in L^{\infty}\left([0, \infty) ; H^{2}(\mathbf{R})\right) \cap C^{0}\left([0, \infty) ; H^{1}(\mathbf{R})\right) \\
\psi(t, x) \in L^{\infty}\left([0, \infty) ; H^{3}(\mathbf{R})\right) \cap C^{0}\left([0, \infty) ; H^{2}(\mathbf{R})\right)
\end{array}\right.
$$

and

$$
\lim _{t \rightarrow \infty}\left(\|\phi(t)\|_{L^{\infty}}+\left\|\phi_{x}(t)\right\|_{L^{\infty}}\right)=0 .
$$

For the corresponding decay estimates, we have the following result.

Theorem 2.2 (Decay estimates) Let $\varphi_{0}(x) \in H^{2}(\mathbf{R}) \cap L^{1}(\mathbf{R})$ and $(\varphi(t, x), \psi(t, x))$ be the unique global solution of the Cauchy problem (2.12)-(2.14) satisfying (1.16) and (1.17), then there exists a positive constant $T$ such that for each fixed $\alpha>4$, we have for any $t \geq 0$ that

$$
\begin{aligned}
& \|\phi(t)\|_{L^{1}} \leq\left\|\phi_{0}(x)\right\|_{L^{1}}+C_{0} \delta \ln (2+t) \\
& (1+t)^{\alpha}\|\phi(t)\|_{1}^{2}+\int_{T}^{t}(1+\tau)^{\alpha}\left\|\partial_{x} \phi(\tau)\right\|^{2} d \tau \\
& \quad \leq D_{1}(E, T)\left(\left\|\phi_{0}(x)\right\|_{1}^{2}+\delta^{2}+\left\|\phi_{0}(x)\right\|_{L^{1}}^{2}\right)(1+t)^{\alpha-\frac{1}{2}} \ln ^{2}(2+t) .
\end{aligned}
$$

Moreover, if we assume further that $\varphi_{0}(x) \in H^{S}(\mathbf{R})$ for each $S \geq 3$, then we have for $\alpha>2 S$ that

$$
\begin{aligned}
& (1+t)^{\alpha+l}\left\|\partial_{x}^{l} \phi(t)\right\|_{1}^{2}+\int_{T}^{t}(1+\tau)^{\alpha+l}\left\|\partial_{x}^{l+1} \phi(\tau)\right\|^{2} d \tau \\
& \leq D_{l+1}(E, T) C_{l+1}\left(\phi_{0}, \delta\right)(1+t)^{\alpha-\frac{1}{2}} \ln ^{12 l-6}(2+t), \quad l=1, \ldots, S-1 .
\end{aligned}
$$

Here $\quad C_{l+1}\left(\phi_{0}, \delta\right)=\left(\left\|\phi_{0}(x)\right\|_{l_{+1}}^{2}+\delta^{2}+\left\|\phi_{0}(x)\right\|_{L^{1}}^{2}\right)\left(1+\left\|\phi_{0}(x)\right\|_{L^{1}}+\left\|\phi_{0}(x)\right\|_{l_{+1}}^{2}\right)^{12 l-8}$, and $D_{1}(E, T), C_{l+1}\left(\varphi_{0}, \delta\right), D_{l+1}(E, T),(l=1, \ldots, S-1)$ are positive constants which depend only on $E$ and $T$.

Remark 2.1 To study the nonlinear stability of planar rarefaction waves for the corresponding multidimensional model, one needs to deal with the Cauchy problem (2.12), (2.13) with zero initial data, i.e.,

$$
\phi(0, x)=\phi_{0}(x) \equiv 0 .
$$

For the Cauchy problem (2.12), (2.13), (2.23), if $\delta$ is sufficiently small, then we have from Theorems 2.1 and 2.2 that such a Cauchy problem admits a unique global solution $(\varphi(t, x), \psi(t, x))$ which satisfies the following decay estimates

$$
\left\{\begin{aligned}
\|\phi(t)\|_{L^{1}} & \leq \tilde{D} \delta \ln (2+t), \\
\left\|\partial_{x}^{l} \phi(t)\right\|_{L^{\infty}} & \leq \tilde{D}_{l} \delta, \quad l \geq 0, \\
\left\|\partial_{x}^{l} \phi(t)\right\|_{L^{\infty}} & \leq \bar{D}_{l}(1+t)^{-\frac{l+1}{2}} \ln ^{6 l}(2+t), \quad l \geq 0 .
\end{aligned}\right.
$$

Here $\tilde{D}, \tilde{D}_{l}$, and $\bar{D}_{l}(l=0,1, \ldots)$ are positive constants independent of $t, x$, and $\delta$ for sufficiently small $\delta>0$. 
We now show that Theorem 1.1 follows immediately from Theorems 2.1 and 2.2. In fact, under the assumptions listed in Theorem 1.1, (1.8) together with (2.2), (2.5), and (2.9) implies that

$$
\begin{aligned}
\left\|\phi_{0}(x)\right\| & \leq\left\|u_{0}(x)-w_{0}^{R}(x)\right\|+\left\|w_{0}^{R}(x)-w^{R}\left(t_{0}, x\right)\right\|+\left\|w^{R}\left(t_{0}, x\right)-w_{0}(x)\right\| \\
& \leq\left\|u_{0}(x)-w_{0}^{R}(x)\right\|+C \delta \\
& \leq\left(D_{1}^{\frac{1}{2}}+C\right) \delta^{\frac{\beta}{2}}:=D_{4} \delta^{\frac{\beta}{2}}
\end{aligned}
$$

and

$$
\begin{aligned}
\left\|\partial_{x}^{k} \phi_{0}(x)\right\| & \leq\left\|\partial_{x}^{k} u_{0}(x)\right\|+\left\|\partial_{x}^{k} w_{0}(x)\right\| \\
& \leq\left(D_{k}\left(1+\delta^{-\gamma}\right)\right)^{\frac{1}{2}}+C \delta \\
& \leq D_{k+4}\left(1+\delta^{-\gamma}\right)^{\frac{1}{2}}, \quad k=1,2 .
\end{aligned}
$$

Here $D_{i}(i=4,5,6)$ are some positive constants which are independent of $x, t$, and $\delta$ for $\delta>0$ sufficiently small.

The above analysis implies that if the assumptions listed in Theorem 1.1 are satisfied, then the conditions listed in Theorems 2.1 and 2.2 are also satisfied. Consequently, we have from Theorems 2.1 and 2.2 that there exists a positive constant $\delta_{0}>0$ such that if $0<\delta \leq \delta_{0}$, the Cauchy problem (1.1), (1.2) admits a unique global solution $(u(t, x)$, $q(t, x))=\left(w(t, x)+\varphi(t, x), \psi(t, x)-\partial_{x} w(t, x)\right)$ and $(\varphi(t, x), \psi(t, x))$ satisfy (2.20)-(2.22).

With (2.20)-(2.22) in hand, we obtain from the Gagliardo-Nirenberg inequality that

$$
\begin{aligned}
\|\phi(t)\|_{L^{p}} & \leq\left\|\phi_{x}(t)\right\|^{\frac{2}{3}\left(1-\frac{1}{p}\right)}\|\phi(t)\|_{L^{1}}^{\frac{p+2}{3 p}} \\
& \leq O(1)(1+t)^{-\frac{1}{2}\left(1-\frac{1}{p}\right)}[\ln (2+t)]^{\frac{7 p-4}{3 p}}, \quad 1 \leq p \leq \infty,
\end{aligned}
$$

and we get from Lemma 2.1 and (2.27) that

$$
\begin{aligned}
\left\|u(t, x)-w^{R}\left(\frac{x}{t}\right)\right\|_{L^{p}} & \leq\|u(t, x)-w(t, x)\|_{L^{p}}+\left\|w(t, x)-w^{R}\left(\frac{x}{t}\right)\right\|_{L^{p}} \\
& \leq O(1)(1+t)^{-\frac{1}{2}\left(1-\frac{1}{p}\right)}[\ln (2+t)]^{\frac{7 p-4}{3 p}}, \quad 1 \leq p \leq \infty .
\end{aligned}
$$

This proves $(1.12)$. (1.13) can be proved similarly. This completes the proof of Theorem 1.1.

\section{The proof of Theorem 2.1}

This section is devoted to proving Theorem 2.1. For this purpose, we first show the local solvability of the Cauchy problem (2.12)-(2.14)

Lemma 3.1 (Local existence) Let $\varphi_{0}(x) \in H^{2}(\mathbf{R})$, then there exists $t_{1}>0$ which depends only on $\left\|\varphi_{0}(x)\right\|_{2}$ such that the Cauchy problem (2.12)-(2.14) admits a unique solution $(\phi(t, x), \psi(t, x)) \in X_{t_{1}}$ satisfying $\left\{\begin{array}{l}\|\phi(t)\| \leq 2\left\|\phi_{0}(x)\right\|_{1}, \\ \left\|\phi_{x}(t)\right\| \leq 2\left\|\phi_{0}(x)\right\|_{1}, \\ \left\|\phi_{x x}(t)\right\| \leq 2\left\|\phi_{0}(x)\right\|_{2} .\end{array}\right.$ 
Here

$$
X_{t_{1}}=\left\{\begin{array}{l|l}
(\phi(t, x), \psi(t, x)) & \begin{array}{l}
\phi(t, x) \in L^{\infty}\left(\left[0, t_{1}\right] ; H^{2}(\mathbf{R})\right) \cap C^{0}\left(\left[0, t_{1}\right] ; H^{1}(\mathbf{R})\right) \\
\psi(t, x) \in L^{\infty}\left(\left[0, t_{1}\right] ; H^{3}(\mathbf{R})\right) \cap C^{0}\left(\left[0, t_{1}\right] ; H^{2}(\mathbf{R})\right)
\end{array}
\end{array}\right\}
$$

with

$$
\|(\phi, \psi)\|_{X_{t_{1}}}=\sup _{0 \leq t \leq t_{1}}\|(\phi(t), \psi(t))\|_{2} .
$$

Lemma 3.1 can be proved by employing the standard method and we omit the details for brevity.

Now suppose that the local solution $(\varphi(t, x), \psi(t, x))$ obtained in Lemma 3.1 has been extended to the time step $t=T \geq t_{1}$, we now turn to deduce certain energy estimates on $(\varphi(t, x), \psi(t, x))$. As mentioned in the introduction, unlike [1], we perform our analysis based on the following a priori assumption

$$
N(T)=\sup _{0 \leq \tau \leq T}\left(\|\phi(\tau)\|_{L^{\infty}}+\left\|\phi_{x}(\tau)\right\|_{L^{\infty}}\right) \leq \eta,
$$

where $\eta$ is a positive constant.

(3.3) together with the fact that $f(u)$ is sufficiently smooth implies that there is a positive constant $L=L(\eta)$ such that

$$
\sum_{i=0}^{4} \max _{|u| \leq\left|u_{+}\right|+\left|u_{-}\right|+\eta}\left|f^{(i)}(u)\right| \leq L .
$$

Based on (3.3) and (3.4), we have

Lemma 3.2 (A priori estimates) Let $\varphi_{0}(x) \in H^{2}(\mathbf{R})$ which verifies the conditions (2.15)-(2.17). Suppose that $(\varphi(t, x), \psi(t, x)) \in X_{T}$ is a solution of the Cauchy problem (2.12)-(2.14) satisfying the a priori assumption (3.3) and (3.4), if

$$
104 \eta L(\eta) \leq 1,
$$

then the following estimates

$$
\begin{aligned}
& \|\phi(t)\|^{2}+\int_{0}^{t}\|\psi(\tau)\|_{1}^{2} d \tau \leq \bar{C}_{0}\left(\left\|\phi_{0}(x)\right\|^{2}+\delta^{2}\right), \\
& \left\|\phi_{x}(t)\right\|^{2}+\int_{0}^{t}\left(\left\|\phi_{x}(\tau)\right\|^{2}+\left\|\psi_{x x}(\tau)\right\|^{2}\right) d \tau \leq \bar{C}_{1}\left(\left\|\phi_{0}(x)\right\|_{1}^{2}+\delta^{2}\right), \\
& \left\|\phi_{x x}(t)\right\|^{2}+\int_{0}^{t}\left(\left\|\phi_{x x}(\tau)\right\|^{2}+\left\|\psi_{x x x}(\tau)\right\|^{2}\right) d \tau \leq \bar{C}_{2}\left(\left\|\phi_{0}(x)\right\|_{2}^{2}+\delta^{2}\right)
\end{aligned}
$$

hold for any $t \in[0, T]$. Here $\bar{C}_{i}(i=0,1,2)$ are positive constants independent of $x$, $T$, and $\delta$ for sufficiently small $\delta>0$.

Proof: We multiply (2.12) and (2.13) by $\varphi$ and $\psi$, respectively, and then add the two resulting identities to obtain

$$
\left(\phi^{2}\right)_{t}+2 \phi(f(w+\phi)-f(w))_{x}+2 \psi^{2}+2 \psi_{x}^{2}-2\left(\psi \psi_{x}\right)_{x}=-2 \phi \frac{f^{\prime \prime \prime}(w)}{f^{\prime \prime}(w)} w_{x}^{2}-2 \psi w_{x x x}
$$


For $0<t<T$, integrating (3.9) over $[0, t] \times \mathbf{R}$ with respect to $t$ and $x$ gives

$$
\begin{aligned}
& \|\phi(t)\|^{2}+2 \int_{0}^{t} \int_{\mathbf{R}} \phi(f(w+\phi)-f(w))_{x} d x d \tau+2 \int_{0}^{t}\|\psi(\tau)\|_{1}^{2} d \tau \\
= & \left\|\phi_{0}(x)\right\|^{2}+2 \int_{0}^{t} \int_{\mathbf{R}}\left(-\phi \frac{f^{\prime \prime \prime}(w)}{f^{\prime \prime}(w)} w_{x}^{2}-\psi w_{x x x}\right) d x d \tau .
\end{aligned}
$$

The second term on the left-hand side of (3.9) is estimated as follows

$$
\begin{aligned}
& 2 \int_{0}^{t} \int_{\mathbf{R}} \phi(f(\phi+w)-f(w))_{x} d x d \tau \\
= & 2 \int_{0}^{t} \int_{\mathbf{R}}\left\{\left(-\int_{w}^{\phi+w} f(s) d s+f(\phi+w) \phi\right)_{x}+\left(f(\phi+w)-f(w)-f^{\prime}(w) \phi\right) w_{x}\right\} d x d \tau \\
= & 2 \int_{0}^{t} \int_{\mathbf{R}}\left(f(\phi+w)-f(w)-f^{\prime}(w) \phi\right) w_{x} d x d \tau \\
= & \int_{0}^{t} \int_{\mathbf{R}} f^{\prime \prime}(w+\theta \phi) w_{x} \phi^{2} d x d \tau \geq 0, \quad 0<\theta<1 .
\end{aligned}
$$

In view of (1.3), the last term on the right-hand side of (3.10) can be controlled as follows

$$
\begin{aligned}
2\left|\int_{0}^{t} \int_{\mathbf{R}} \phi \frac{f^{\prime \prime \prime}(w)}{f^{\prime \prime}(w)} w_{x}^{2} d x d \tau\right| & \leq \frac{a}{2} \int_{0}^{t} \int_{\mathbf{R}} w_{x} \phi^{2} d x d \tau+\frac{2}{a} \int_{0}^{t} \int_{\mathbf{R}}^{t}\left|\frac{f^{\prime \prime \prime}(w)}{f^{\prime \prime}(w)}\right|^{2}\left(w_{x}\right)^{3} d x d \tau \\
& \leq \frac{a}{2} \int_{0}^{t} \int_{\mathbf{R}} w_{x} \phi^{2} d x d \tau+O(1) \int_{0}^{t}\left\|w_{x}(\tau)\right\|_{L^{3}}^{3} d \tau
\end{aligned}
$$

and

$$
2\left|\int_{0}^{t} \int_{\mathbf{R}} \psi w_{x x x} d x d \tau\right| \leq \int_{0}^{t}\|\psi(\tau)\|^{2} d \tau+\int_{0}^{t}\left\|w_{x x x}(\tau)\right\|^{2} d \tau .
$$

Substituting (3.11)-(3.13) into (3.10) and (3.6) follows immediately from Lemma 2.1.

Next, we show that the estimate (3.7) holds if (3.5) is satisfied. To this end, we have by differentiating (2.12) and (2.13) with respect to $x$ once, multiplying the resulting identities by $\varphi_{x}$ and $\psi_{x}$, respectively, and adding these two identities yield that

$$
\phi_{x} \phi_{x t}+\phi_{x}(f(w+\phi)-f(w))_{x x}+\psi_{x}^{2}+\psi_{x x}^{2}-\left(\psi_{x x} \psi_{x}\right)_{x}=-\phi_{x}\left\{\frac{f^{\prime \prime \prime}(w)}{f^{\prime \prime}(w)} w_{x}^{2}\right\}_{x}-\psi_{x} w_{x x x x} .
$$

Noticing that

$$
\begin{aligned}
\phi_{x}(f(w+\phi)-f(w))_{x x}= & \frac{3}{2} f^{\prime \prime}(w+\phi) w_{x} \phi_{x}^{2}+\frac{1}{2} f^{\prime \prime}(w+\phi) \phi_{x}^{3}+\left(f^{\prime \prime}(w+\phi)-f^{\prime \prime}(w) w_{x}^{2} \phi_{x}\right. \\
& +\left(f^{\prime}(w+\phi)-f^{\prime}(w)\right) w_{x x} \phi_{x}+\frac{1}{2}\left[f^{\prime}(w+\phi) \phi_{x}^{2}\right]_{x}
\end{aligned}
$$


we have from (3.14) and (3.15) that

$$
\begin{aligned}
& \left(\phi_{x}^{2}\right)_{t}+3 f^{\prime \prime}(w+\phi) w_{x} \phi_{x}^{2}+2 \psi_{x}^{2}+2 \psi_{x x}^{2}+(\cdots)_{x} \\
= & -f^{\prime \prime}(w+\phi) \phi_{x}^{3}-2\left(f^{\prime \prime}(w+\phi)-f^{\prime \prime}(w)\right) w_{x}^{2} \phi_{x}-2\left(f^{\prime}(w+\phi)-f^{\prime}(w)\right) w_{x x} \phi_{x} \\
& -2 \phi_{x}\left\{\frac{f^{\prime \prime \prime}(w)}{f^{\prime \prime}(w)} w_{x}^{2}\right\}_{x}-2 \psi_{x} w_{x x x x} .
\end{aligned}
$$

Here and in the rest of this article, $(\ldots)_{x}$ denotes some terms which vanish after integration with respect to $x$ over $x \in \mathbf{R}$.

On the other hand, we have from (2.13) that

$$
\phi_{x}^{2}+w_{x x x}^{2}=\psi^{2}+2 \psi_{x}^{2}+\psi_{x x}^{2}-2 \phi_{x} w_{x x x}-2\left(\psi \psi_{x}\right)_{x} .
$$

Adding (3.16) to (3.17) yields

$$
\left(\phi_{x}^{2}\right)_{t}+3 f^{\prime \prime}(w+\phi) w_{x} \phi_{x}^{2}+\phi_{x}^{2}+\psi_{x x}^{2}+w_{x x x}^{2}+(\cdots)_{x}=\psi^{2}+F_{1}
$$

where

$$
\begin{aligned}
F_{1}= & -f^{\prime \prime}(w+\phi) \phi_{x}^{3}-2\left(f^{\prime \prime}(w+\phi)-f^{\prime \prime}(w)\right) w_{x}^{2} \phi_{x}-2\left(f^{\prime}(w+\phi)-f^{\prime}(w)\right) w_{x x} \phi_{x} \\
& -2 \psi_{x} w_{x x x x}-2 \phi_{x} w_{x x x}-2 \phi_{x}\left\{\frac{f^{\prime \prime \prime}(w)}{f^{\prime \prime}(w)} w_{x}^{2}\right\}_{x} .
\end{aligned}
$$

Integrating (3.18) with respect to $t$ and $x$ over $[0, t] \times \mathbf{R}$ yields

$$
\left\|\phi_{x}(t)\right\|^{2}+\int_{0}^{t}\left(\left\|\psi_{x x}(\tau)\right\|^{2}+\left\|\phi_{x}(\tau)\right\|^{2}\right) d \tau \leq\left\|\phi_{0}^{\prime}(x)\right\|^{2}+\int_{0}^{t} \int_{\mathbf{R}}\left(\psi^{2}+F_{1}\right) d x d \tau .
$$

From (3.6), it is easy to see that

$$
\int_{0}^{t} \int_{\mathbf{R}} \psi^{2}(\tau, x) d x d \tau \leq \bar{C}_{0}\left(\left\|\phi_{0}(x)\right\|^{2}+\delta^{2}\right) .
$$

For the corresponding terms appearing in $F_{1}$, we have from Lemma 2.1, the a priori assumption (3.3) and (3.4) that

$$
\begin{aligned}
&\left|\int_{0}^{t} \int_{\mathbf{R}} f^{\prime \prime}(w+\phi) \phi_{x}^{3} d x d \tau\right| \leq L N(t) \int_{0}^{t}\left\|\phi_{x}(\tau)\right\|^{2} d \tau \\
& 2\left|\int_{0}^{t} \int_{\mathbf{R}}\left(f^{\prime \prime}(w+\phi)-f^{\prime \prime}(w)\right) w_{x}^{2} \phi_{x} d x d \tau\right| \leq 4 L \int_{0}^{t}\left\|\phi_{x}(\tau)\right\|\left\|w_{x}(\tau)\right\|_{L^{4}}^{2} d \tau \\
& \leq \frac{1}{8} \int_{0}^{t}\left\|\phi_{x}(\tau)\right\|^{2} d \tau+O(1) \delta^{2}, \\
& 2\left|\int_{0}^{t} \int_{\mathbf{R}}\left(f^{\prime}(w+\phi)-f^{\prime}(w)\right) \phi_{x} w_{x x} d x d \tau\right| \leq 4 L \int_{0}^{t}\left\|\phi_{x}(\tau)\right\|\left\|w_{x x}(\tau)\right\| d \tau \\
& \leq \frac{1}{8} \int_{0}^{t}\left\|\phi_{x}(\tau)\right\|^{2} d \tau+O(1) \delta^{2} .
\end{aligned}
$$


Similarly, we can get that

$$
\begin{aligned}
& 2\left|\int_{0}^{t} \int_{\mathbf{R}} \phi_{x} w_{x x x} d x d \tau\right| \leq \frac{1}{8} \int_{0}^{t}\left\|\phi_{x}(\tau)\right\|^{2} d \tau+O(1) \delta^{2}, \\
& 2\left|\int_{0}^{t} \int_{\mathbf{R}} \phi_{x}\left\{\frac{f^{\prime \prime \prime}(w)}{f^{\prime \prime}(w)} w_{x}^{2}\right\} d x d \tau\right| \leq \frac{1}{8} \int_{0}^{t}\left\|\phi_{x}(\tau)\right\|^{2} d \tau+O(1) \delta^{2}, \\
& 2\left|\int_{0}^{t} \int_{\mathbf{R}} \psi_{x} w_{x x x x} d x d \tau\right| \leq \int_{0}^{t}\left\|\psi_{x}(\tau)\right\|^{2} d \tau+O(1) \delta^{2} \leq O(1)\left(\left\|\phi_{0}(x)\right\|^{2}+\delta^{2}\right) .
\end{aligned}
$$

Putting (3.22)-(3.27) together implies

$$
\int_{0}^{t} \int_{\mathbf{R}} F_{1}(\tau, x) d x d \tau \leq\left(L N(t)+\frac{1}{2}\right) \int_{0}^{t}\left\|\phi_{x}(\tau)\right\|^{2} d \tau+O(1)\left(\left\|\phi_{0}(x)\right\|^{2}+\delta^{2}\right) .
$$

Inserting (3.21) and (3.28) into (3.20), we can deduce that

$$
\left\|\phi_{x}(t)\right\|^{2}+\int_{0}^{t}\left(\left\|\psi_{x x}(\tau)\right\|^{2}+\left(\frac{1}{2}-L N(T)\right)\left\|\phi_{x}(\tau)\right\|^{2}\right) d \tau \leq O(1)\left(\left\|\phi_{0}(x)\right\|_{1}^{2}+\delta^{2}\right) .
$$

Noticing that (3.5) implies that

$$
\frac{1}{2}-L N(t) \geq \frac{51}{104}
$$

and (3.7) follows immediately from (3.29) and the above inequality.

Finally, we deduce (3.8). For this purpose, we differentiate (2.12) and (2.13) with respect to $x$ twice, multiply them by $\varphi_{x x}$ and $\psi_{x x}$, respectively, and add the two resulting equations to get that

$$
\left(\phi_{x x}^{2}\right)_{t}+5 f^{\prime \prime}(w+\phi) w_{x} \phi_{x x}^{2}+2 \psi_{x x}^{2}+2 \psi_{x x x}^{2}+(\cdots)_{x}=F_{2}+2 \phi_{x x} w_{x x x x}
$$

where

$$
\begin{aligned}
F_{2}= & -5 f^{\prime \prime}(w+\phi) \phi_{x x}^{2} \phi_{x}-2\left(f^{\prime}(w+\phi)-f^{\prime}(w)\right) w_{x x x} \phi_{x x}-6\left(f^{\prime \prime}(w+\phi)-f^{\prime \prime}(w)\right) w_{x x} w_{x} \phi_{x x} \\
& -2\left(f^{\prime \prime \prime}(w+\phi)-f^{\prime \prime \prime}(w)\right) w_{x}^{3} \phi_{x x}-6 f^{\prime \prime}(w+\phi) w_{x x} \phi_{x x} \phi_{x}-6 f^{\prime \prime \prime}(w+\phi) w_{x}^{2} \phi_{x x} \phi_{x} \\
& -6 f^{\prime \prime \prime}(w+\phi) w_{x} \phi_{x x} \phi_{x}^{2}-2 f^{\prime \prime \prime}(w+\phi) \phi_{x x} \phi_{x}^{3} \\
& -2 \phi_{x x} w_{x x x x}-2 \phi_{x x}\left\{\frac{f^{\prime \prime \prime}(w)}{f^{\prime \prime}(w)} w_{x}^{2}\right\}_{x x}-2 \psi_{x x} w_{x x x x x} .
\end{aligned}
$$

On the other hand, we have from (2.13) that

$$
\phi_{x x}^{2}+w_{x x x x}^{2}=\psi_{x}^{2}+2 \psi_{x x}^{2}+\psi_{x x x}^{2}-2 \phi_{x x} w_{x x x x}-2\left(\psi_{x} \psi_{x x}\right)_{x}
$$

Combining (3.30) with (3.32) yields

$$
\left(\phi_{x x}^{2}\right)_{t}+5 f^{\prime \prime}(w+\phi) w_{x} \phi_{x x}^{2}+\phi_{x x}^{2}+\psi_{x x x}^{2}+\psi_{x x x x}^{2}+(\cdots)_{x}=\psi_{x}^{2}+F_{2}
$$

Integrating (3.33) with respect to $t$ and $x$ over $[0, t] \times \mathbf{R}$, we can deduce that

$$
\left\|\phi_{x x}(t)\right\|^{2}+\int_{0}^{t}\left(\left\|\psi_{x x x}(\tau)\right\|^{2}+\left\|\phi_{x x}(\tau)\right\|^{2}\right) d \tau \leq\left\|\phi_{0}^{\prime \prime}(x)\right\|^{2}+\int_{0}^{t} \int_{\mathbf{R}}\left(\psi_{x}^{2}+F_{2}\right) d x d \tau .
$$


Now we turn to deal with the terms appeared on the right-hand side of (3.34). First, from (3.6), it is easy to see that

$$
\int_{0}^{t} \int_{\mathbf{R}} \psi_{x}^{2}(\tau, x) d x d \tau \leq \bar{C}_{0}\left(\left\|\phi_{0}(x)\right\|^{2}+\delta^{2}\right) .
$$

As to the terms appearing in $F_{2}$, we have from Lemma 2.1, the a priori assumption (3.3), (3.4), (3.6), and (3.7) that

$$
\begin{aligned}
& 5\left|\int_{0}^{t} \int_{\mathbf{R}} f^{\prime \prime}(w+\phi) \phi_{x x}^{2} \phi_{x} d x d \tau\right| \leq 5 L N(t) \int_{0}^{t}\left\|\phi_{x x}(\tau)\right\|^{2} d \tau, \\
&\left|2 \int_{0}^{t} \int_{\mathbf{R}}\left(f^{\prime}(w+\phi)-f^{\prime}(w)\right) w_{x x x} \phi_{x x} d x d \tau\right| \leq 4 L \int_{0}^{t}\left\|\phi_{x x}(\tau)\right\|\left\|w_{x x x}(\tau)\right\| d \tau \\
& \leq \frac{1}{8} \int_{0}^{t}\left\|\phi_{x x}(\tau)\right\|^{2} \mathrm{~d} \tau+O(1) \delta^{2},
\end{aligned}
$$

$6\left|\int_{0}^{t} \int_{\mathbf{R}}\left(f^{\prime \prime}(w+\phi)-f^{\prime \prime}(w)\right) w_{x} w_{x x} \phi_{x x} d x d \tau\right| \leq O(1) L \int_{0}^{t}\left\|\phi_{x x}(\tau)\right\|\left\|w_{x}(\tau)\right\|_{L^{\infty}}\left\|w_{x x}(\tau)\right\| d \tau$

$$
\leq \frac{1}{8} \int_{0}^{t}\left\|\phi_{x x}(\tau)\right\|^{2} d \tau+O(1) \delta^{4}
$$

$$
\begin{aligned}
2\left|\int_{0}^{t} \int_{\mathbf{R}}\left(f^{\prime \prime \prime}(w+\phi)-f^{\prime \prime \prime}(w)\right) w_{x}^{3} \phi_{x x} d x d \tau\right| & \leq 4 L \int_{0}^{t}\left\|\phi_{x x}(\tau)\right\|\left\|w_{x}(\tau)\right\|_{L^{6}}^{3} d x d \tau \\
& \leq \frac{1}{8} \int_{0}^{t}\left\|\phi_{x x}(\tau)\right\|^{2} d \tau+O(1) \delta^{6},
\end{aligned}
$$

$$
\begin{aligned}
6 \mid \int_{0}^{t} \int_{\mathbf{R}}\left(f^{\prime \prime}(w+\phi) w_{x x} \phi_{x x} \phi_{x} d x d \tau \mid\right. & \leq 6 L N(t) \int_{0}^{t}\left\|\phi_{x x}(\tau)\right\|\left\|w_{x x}(\tau)\right\| d \tau \\
& \leq 3 L N(t) \int_{0}^{t}\left\|\phi_{x x}(\tau)\right\|^{2} d \tau+O(1) \delta^{2} .
\end{aligned}
$$

Similarly, we can get that

$$
\begin{aligned}
2 \mid \int_{0}^{t} \int_{\mathbf{R}}\left(f^{\prime \prime \prime}(w+\phi) \phi_{x}^{3} \phi_{x x} d x d \tau \mid\right. & \leq 2 L(N(t))^{2} \int_{0}^{t}\left\|\phi_{x x}(\tau)\right\|\left\|\phi_{x}(\tau)\right\| d \tau \\
& \leq L N(t) \int_{0}^{t}\left\|\phi_{x x}(\tau)\right\|^{2} d \tau+C(N(t))\left(\left\|\phi_{0}(x)\right\|_{1}^{2}+\delta^{2}\right),
\end{aligned}
$$




$$
\begin{aligned}
& 6 \mid \int_{0}^{t} \int_{\mathbf{R}}\left(f^{\prime \prime \prime}(w+\phi) w_{x}^{2} \phi_{x x} \phi_{x} d x d \tau \mid \leq 3 L N(t) \int_{0}^{t}\left\|\phi_{x x}(\tau)\right\|^{4} d \tau+O(1) \delta^{2},\right. \\
& 6 \mid \int_{0}^{t} \int_{\mathbf{R}}\left(f^{\prime \prime \prime}(w+\phi) w_{x} \phi_{x}^{2} \phi_{x x} d x d \tau \mid \leq 6 L N(t) \int_{0}^{t}\left\|w_{x}(\tau)\right\|_{L^{\infty}}\left\|\phi_{x}(\tau)\right\|\left\|\phi_{x x}(\tau)\right\| d \tau\right. \\
& 2\left|\int_{0}^{t} \int_{\mathbf{R}}^{t} w_{x x x x} \phi_{x x} d x d \tau\right| \leq \frac{1}{8} \int_{0}^{t}\left\|\int_{0}^{t}\right\| \phi_{x x}(\tau) \|^{2} d \tau+C(N(t))\left(\left\|\phi_{0}(x)\right\|_{1}^{2}+\delta^{2}\right), \\
& 2 \mid \int_{0}^{t} \int_{\mathbf{R}} \phi_{x x}\left\{\frac{f^{\prime \prime \prime}(w)}{f^{\prime \prime}(w)} w_{x}^{2}\right\} \\
& x x
\end{aligned}
$$

and

$$
2\left|\int_{0}^{t} \int_{\mathbf{R}} w_{x x x x x} \psi_{x x} d x d \tau\right| \leq \frac{1}{2} \int_{0}^{t}\left\|\psi_{x x}(\tau)\right\|^{2} d \tau+O(1) \delta^{2} .
$$

Substituting (3.35)-(3.46) into (3.34) and noticing that (3.5) implies that

$$
13 L N(t) \leq \frac{1}{8}
$$

we can deduce that (3.8) follows immediately. Thus Lemma 3.2 is proved.

Now we turn to prove Theorem 2.1. First, from the local solvability result Lemma 3.1 , we know that there is a positive constant $t_{1}>0$ such that the Cauchy problem (2.12)-(2.14) admits a unique solution $(\phi(t, x), \psi(t, x)) \in X_{t_{1}}$ which satisfies

$$
\left\{\begin{aligned}
\|\phi(t)\| & \leq 2\left\|\phi_{0}(x)\right\| \\
\left\|\phi_{x}(t)\right\| & \leq 2\left\|\phi_{0}(x)\right\|_{1}^{\prime} \\
\left\|\phi_{x x}(t)\right\| & \leq 2\left\|\phi_{0}(x)\right\|_{2}
\end{aligned}\right.
$$

for $0 \leq t \leq t_{1}$.

Now since the initial perturbation is assumed to satisfy (2.15)-(2.17), we have from (3.47) and the Gagliardo-Nirenberg inequality that the following estimates

$$
\left\{\begin{array}{l}
\|\phi(t)\|_{L^{\infty}} \leq D\|\phi(t)\|^{\frac{3}{4}}\left\|\phi_{x x}(t)\right\|^{\frac{1}{4}} \leq 2 D\left(D_{1}\right)^{\frac{3}{8}}\left(D_{3,1}\right)^{\frac{1}{8}} \frac{\frac{3 \beta-\gamma}{8}}{\delta^{2}}\left(1+\delta^{\gamma}\right)^{\frac{1}{8}} \\
\left\|\phi_{x}(t)\right\|_{L^{\infty}} \leq D\|\phi(t)\|^{\frac{1}{4}}\left\|\phi_{x x}(t)\right\|^{\frac{3}{4}} \leq 2 D\left(D_{1}\right)^{\frac{1}{8}}\left(D_{3,1}\right)^{\frac{3}{8}} \frac{\beta-3 \gamma}{8}\left(1+\delta^{\gamma}\right)^{\frac{3}{8}}
\end{array}\right.
$$

hold for any $t \in\left[0, t_{1}\right]$. Here $D>0$ is the constant appearing in the Gagliardo-Nirenberg inequality and $D_{3,1}=\sum_{1 \leq i \leq 3} D_{i}$.

(3.48) implies that there exists a positive constant $D_{7}>0$ which is independent of $x$, $t$, and $\delta$ for sufficiently small $\delta>0$ such that 


$$
\|\phi(t)\|_{L^{\infty}}+\left\|\phi_{x}(t)\right\|_{L^{\infty}} \leq D_{7} \delta \frac{\beta-3 \gamma}{8}
$$

holds for any $t \in\left[0, t_{1}\right]$.

Thus if $\beta>3 \gamma$, we can easily deduce that there exists $\delta_{1}>0$ sufficiently small such that if $0<\delta \leq \delta_{1}$, we have for $0 \leq t \leq t_{1}$ that

$$
104 D_{7} \delta \frac{\beta-3 \gamma}{8} L\left(D_{7} \delta \frac{\beta-3 \gamma}{8}\right) \leq 1
$$

which means that the conditions listed in Lemma 3.2 hold with $T=t_{1}$, $\eta=D_{7} \delta \frac{\beta-3 \gamma}{8}$ and thus we have from Lemma 3.2 that

$$
\left\{\begin{array}{l}
\|\phi(t)\|^{2} \leq \bar{C}_{1}\left(\left\|\phi_{0}(x)\right\|^{2}+\delta^{2}\right), \\
\left\|\phi_{x}(t)\right\|^{2} \leq \bar{C}_{2}\left(\left\|\phi_{0}(x)\right\|_{1}^{2}+\delta^{2}\right) \\
\left\|\phi_{x x}(t)\right\|^{2} \leq \bar{C}_{3}\left(\left\|\phi_{0}(x)\right\|_{2}^{2}+\delta^{2}\right)
\end{array}\right.
$$

hold for $t \in\left[0, t_{1}\right]$.

Now take $\varphi\left(t_{1}, x\right)$ as initial data, we have from the local existence result Lemma 3.1 again that there exists a positive constant $t_{2}>0$ such that the solution $(\varphi(t, x), \psi(t, x))$ of Cauchy problem (2.12)-(2.14) obtained above can be extended to the time step $t=$ $t_{1}+t_{2}$ and $(\varphi(t, x), \psi(t, x))$ satisfies

$$
\left\{\begin{array}{l}
\|\phi(t)\|^{2} \leq 4 \bar{C}_{1}\left(\left\|\phi_{0}(x)\right\|^{2}+\delta^{2}\right) \\
\left\|\phi_{x}(t)\right\|^{2} \leq 4 \bar{C}_{2}\left(\left\|\phi_{0}(x)\right\|_{1}^{2}+\delta^{2}\right) \\
\left\|\phi_{x x}(t)\right\|^{2} \leq 4 \bar{C}_{3}\left(\left\|\phi_{0}(x)\right\|_{2}^{2}+\delta^{2}\right)
\end{array}\right.
$$

for $t \in\left[t_{1}, t_{1}+t_{2}\right]$.

Notice that (3.51) together with (3.50) implies that (3.51) holds for all $t \in\left[0, t_{1}+t_{2}\right]$ and consequently we have from the assumptions (2.15)-(2.17) imposed on the initial perturbation, (3.51) and the Gagliardo-Nirenberg inequality that

$$
\left\{\begin{array}{l}
\|\phi(t)\|_{L^{\infty}} \leq D\|\phi(t)\|^{\frac{3}{4}}\left\|\phi_{x x}(t)\right\|^{\frac{1}{4}} \leq 4 D\left(\bar{C}_{1} D_{1}\right)^{\frac{3}{8}}\left(D_{3,2}\right)^{\frac{1}{8}} \frac{3 \beta}{8}\left(1+\delta^{-\gamma}\right)^{\frac{1}{8}}, \\
\left\|\phi_{x}(t)\right\|_{L^{\infty}} \leq D\|\phi(t)\|^{\frac{1}{4}}\left\|\phi_{x x}(t)\right\|^{\frac{3}{4}} \leq 4 D\left(\bar{C}_{1} D_{1}\right)^{\frac{1}{8}}\left(D_{3,2}\right)^{\frac{3}{8}} \delta^{\frac{\beta}{8}}\left(1+\delta^{-\gamma}\right)^{\frac{3}{8}}
\end{array}\right.
$$

hold for any $t \in\left[0, t_{1}+t_{2}\right]$. Here $D_{3,2}=\sum_{1 \leq i \leq 3} \bar{C}_{i} D_{i}$.

(3.52) implies that there exists a positive constant $D_{8}>0$ which are independent of $x$, $t$ and $\delta>0$ for sufficiently small $\delta>0$ such that

$$
\|\phi(t)\|_{L^{\infty}}+\left\|\phi_{x}(t)\right\|_{L^{\infty}} \leq D_{8} \delta \frac{\beta-3 \gamma}{8}
$$

holds for any $t \in\left[0, t_{1}+t_{2}\right]$. 
Having obtained (3.53), we can choose by repeating the argument used above to show that there exists a sufficiently small positive constant $\delta_{2}>0$ such that for 0 $<\delta_{\leq} \delta_{2}$, we have for $0 \leq t \leq t_{1}+t_{2}$ that

$$
104 D_{8} \delta \frac{\beta-3 \gamma}{8} L\left(D_{8} \delta \frac{\beta-3 \gamma}{8}\right) \leq 1
$$

which means that the conditions listed in Lemma 3.2 hold for $T=t_{1}+t_{2}$ and $\eta=D_{8} \delta \frac{\beta-3 \gamma}{8}$ and thus we have from Lemma 3.2 that (3.50) holds for any $t \in\left[0, t_{1}+\right.$ $\left.t_{2}\right]$.

Now take $\varphi\left(t_{1}+t_{2}, x\right)$ as initial data, we can employ Lemma 3.1 once more to deduce that $(\varphi(t, x), \psi(t, x))$ can be extended to the time step $t=t_{1}+2 t_{2}$ and (3.51) holds for $t \in\left[t_{1}+t_{2}, t_{1}+2 t_{2}\right]$. Since the constant $C$ in (3.50) is independent of $t$, we can thus repeat the above process to extend $(\varphi(t, x), \psi(t, x))$ to a global one.

The above analysis tells us that if $0<\delta \leq \delta_{0}$ with $\delta_{0}=\min \left\{\delta_{1}, \delta_{2}\right\}$, then the Cauchy problem (2.15)-(2.17) admits a unique global solution $(\varphi(t, x), \psi(t, x))$ which satisfies

$$
\|\phi(t)\|_{2}^{2}+\int_{0}^{t}\left(\|\psi(\tau)\|_{3}^{2}+\left\|\phi_{x}(\tau)\right\|_{1}^{2}\right) d \tau \leq O(1)\left(\left\|\phi_{0}\right\|_{2}^{2}+\delta^{2}\right), \quad t \geq 0 .
$$

Having obtained (3.54), the proof of (2.19) is standard, we thus omit the details for brevity.

\section{The proof of Theorem 2.2}

The main purpose of this section is devoted to deducing the corresponding convergence rates of the global solution $(\varphi(t, x), \psi(t, x))$ of the Cauchy problem (2.12)-(2.14) to the rarefaction waves $\left(w^{R}\left(\left(\frac{x}{t}\right),-\partial_{x} w^{R}\left(\frac{x}{t}\right)\right)\right.$ under the assumptions that the Cauchy problem (2.12)-(2.14) admits a unique global solution $(\varphi(t, x), \psi(t, x))$ which satisfies (1.16), (1.17).

Before stating our main result, we first deduce certain estimates on $(\varphi(t, x), \psi(t, x))$ based on the assumption (1.16)

Lemma 4.1 Let $\varphi_{0}(x) \in H^{S}(\mathbf{R})$ and $(\varphi(t, x), \psi(t, x))$ be the global solution of the Cauchy problem (2.12)-(2.14). If we assume further that $(\varphi(t, x), \psi(t, x))$ satisfies (1.16), then for each fixed $T>0$, there exists a positive constant $C_{k}(E, T)>0$ such that

$$
\left\|\partial_{x}^{l} \phi(T)\right\|_{1}^{2} \leq C_{l}(E, T)\left(\left\|\phi_{0}(x)\right\|_{l+1}^{2}+\delta^{2}\right), \quad l=0, \ldots, S-1 .
$$

Lemma 4.1 can be proved by employing the standard energy estimates and thus we omit the details for brevity.

Remark 4.1 If the constant $E$ in (1.16) is assumed to be sufficiently small, then the constant $C(E, T)$ can be chosen independent of $T>0$. This fact follows easily from the proof of Lemma 3.2.

The following lemma is concerned with the $L^{1}$ estimate on $\varphi(t, x)$.

Lemma 4.2 Under the assumptions listed in Lemma 4.1, if we assume further that $\varphi_{0}$ $(x) \in L^{1}(\mathbf{R})$, then we can get that 


$$
\|\phi(t)\|_{L^{1}} \leq\left\|\phi_{0}(x)\right\|_{L^{1}}+C_{0} \delta \ln (2+t),
$$

where $C_{0}$ is independent of $t, x$, and $\delta$.

The proof of Lemma 4.2 is similar to that of $[1,4]$ and thus we omit the details for brevity. With the above preliminaries in hand, we now state the main results of this section

Theorem 4.1 (Decay estimates) Let $\varphi_{0}(x) \in H^{S}(\mathbf{R}) \cap L^{1}(\mathbf{R})$ and $(\varphi(t, x), \psi(t, x))$ be the unique global solution of the Cauchy problem (2.12)-(2.14) satisfying (1.16) and (1.17), then there exists a positive constant $T>0$ which is chosen sufficiently large such that for each $\alpha>2 S, l=1, \ldots, S-1$, we have for each $t \geq T$ that

$$
\begin{aligned}
& (1+t)^{\alpha}\|\phi(t)\|_{1}^{2}+\int_{T}^{t}(1+\tau)^{\alpha}\left\|\partial_{x} \phi(\tau)\right\|^{2} d \tau \\
\leq & D_{1}(E, T)\left(\left\|\phi_{0}(x)\right\|_{1}^{2}+\delta^{2}+\left\|\phi_{0}(x)\right\|_{L^{1}}^{2}\right)(1+t)^{\alpha-\frac{1}{2}} \ln ^{2}(2+t), \\
& (1+t)^{\alpha+l}\left\|\partial_{x}^{l} \phi(t)\right\|_{1}^{2}+\int_{T}^{t}(1+\tau)^{\alpha+l}\left\|\partial_{x}^{l+1} \phi(\tau)\right\|^{2} d \tau \\
\leq & D_{l+1}(E, T) C_{l+1}\left(\phi_{0}, \delta\right)(1+t)^{\alpha-\frac{1}{2}} \ln ^{12 l-6}(2+t) .
\end{aligned}
$$

Here $D_{l}, D_{l}(l=1, \ldots, S)$ are positive constants defined in (2.21) and (2.22), respectively.

Remark 4.2 If $f(u)=\frac{1}{2} u^{2}$, (4.2) can be improved to

$$
\|\phi(t)\|_{L^{1}} \leq\left\|\phi_{0}(x)\right\|_{L^{1}}+C_{0} \delta
$$

and consequently we can show that the decay estimates (2.20)-(2.22) can be improved such that the term $\ln (2+t)$ in the right-hand side can be replaced by 1 .

Theorem 4.1 will be proved by the method of mathematical induction. For this purpose, we first choose $\bar{\eta}>0$ sufficiently small such that

$$
(k+2) M \bar{\eta} \leq \frac{1}{64}, \quad M=\max _{0 \leq j \leq S|u| \leq \bar{\eta}_{+}\left|u_{-}+\right| u_{+} \mid}\left|f^{(j+1)}(u)\right| .
$$

On the other hand, the assumptions in Theorem 4.1 tell us that $\varphi(t, x)$ satisfies (1.17).

From which we can deduce that there exists a sufficiently large constant $T>0$ such that

$$
\begin{gathered}
\|\phi(t)\|_{L^{\infty}}+\left\|\phi_{x}(t)\right\|_{L^{\infty}} \leq \bar{\eta}, \quad \forall t \geq T, \\
16\left(\alpha+S+4(k+2) M\left(C_{\infty}+\bar{\eta}\right)\right) \leq 1+T,
\end{gathered}
$$

where $C_{\infty}$ is the constant in (2.8) for $p=\infty$.

Now we turn to show (4.3) and (4.4) for $l=1$. To this end, we have by adding (3.9) and (3.18) together, multiplying the resulting identity by $(1+t)^{\alpha}$, and integrating the final results with respect to $t$ and $x$ over $[T, t) \times \mathbf{R}$ to deduce that 


$$
\begin{aligned}
& (1+t)^{\alpha}\|\phi(t)\|_{1}^{2}+\int_{T}^{t}(1+\tau)^{\alpha}\left(\left\|\phi_{x}(\tau)\right\|^{2}+\|\psi(\tau)\|_{2}^{2}\right) d \tau \\
\leq & \underbrace{(1+T)^{\alpha}\|\phi(T)\|_{1}^{2}}_{H_{1}}+\alpha \underbrace{\int_{T}^{t}(1+\tau)^{\alpha-1}\|\phi(\tau)\|^{2} d \tau}_{H_{2}}+\alpha \underbrace{\int_{T}^{t}(1+\tau)^{\alpha-1}\left\|\phi_{x}(\tau)\right\|^{2} d \tau}_{H_{3}} \\
& +\underbrace{\int_{T}^{t} \int_{\mathbf{R}}(1+\tau)^{\alpha}\left\{F_{1}-2 \phi_{x}\left(\frac{f^{\prime \prime \prime}(w)}{f^{\prime \prime}(w)} w_{x}^{2}\right)_{x}-2 \psi_{x} w_{x x x x}\right\} d x d \tau .}_{H_{4}}
\end{aligned}
$$

Now we turn to estimate $H_{1}-H_{4}$ term by term. First (4.1) tells us that

$$
H_{1} \leq C_{1}(E, T)\left(\left\|\phi_{0}(x)\right\|_{1}^{2}+\delta^{2}\right)
$$

and (4.7) implies that

$$
H_{3} \leq \frac{1}{16} \int_{T}^{t}(1+\tau)^{\alpha}\left\|\phi_{x}(\tau)\right\|^{2} d \tau
$$

As to $H_{2}$ and $H_{4}$, from (4.2), we have by repeating the argument used in [1] that

$$
H_{2} \leq \frac{1}{16} \int_{T}^{t}(1+\tau)^{\alpha}\left\|\phi_{x}(\tau)\right\|^{2} d \tau+O(1)(1+t)^{\alpha-\frac{1}{2}}\left(\delta \ln (2+t)+\left\|\phi_{0}(x)\right\|_{L^{1}}\right)^{2}(4
$$

and

$$
H_{4} \leq\left(\frac{5}{16}+M \eta\right) \int_{T}^{t}(1+\tau)^{\alpha}\left(\left\|\phi_{x}(\tau)\right\|^{2}+\left\|\psi_{x}(\tau)\right\|_{1}^{2}\right) d \tau+O(1)(1+t)^{\alpha-1}\left(\delta \ln (2+t)+\left\|\phi_{0}(x)\right\|_{L^{1}}\right) .
$$

Substituting (4.9)-(4.12) into (4.8), we can deduce (4.3) holds immediately from (4.6) and (4.7).

On the other hand, from (3.16) and (3.33), we can get by employing the same argument that

$$
\begin{aligned}
& (1+t)^{\alpha+1}\left\|\phi_{x}(t)\right\|_{1}^{2}+\int_{T}^{t}(1+\tau)^{\alpha+1}\left(\left\|\phi_{x x}(\tau)\right\|^{2}+\left\|\psi_{x}(\tau)\right\|_{2}^{2}\right) d \tau \\
\leq & (1+T)^{\alpha+1}\left\|\phi_{x}(T)\right\|_{1}^{2}+(\alpha+1) \int_{T}^{t}(1+\tau)^{\alpha}\left\|\phi_{x}(\tau)\right\|_{1}^{2} d \tau \\
& +\int_{T}^{t} \int_{R}(1+\tau)^{\alpha+1}\left(F_{1}+2 \phi_{x} w_{x x x}+F_{2}\right) d x d \tau .
\end{aligned}
$$

With (4.13) and (4.3) in hand, (4.4) with $l=1$ can be proved by the same argument. The above analysis implies that (4.3) and (4.4) for $l=1$ hold true.

Now suppose that (4.4) holds for $l=1,2, \ldots, k-1$ for some $k \geq 2$, that is, there exist some positive constants $C_{l+1}, D_{l+1}(l=1, \ldots, k-1)$ independent of $t, x$, and $\delta$ such that 


$$
\left.(1+t)^{\alpha+l}\left\|\partial_{x}^{l} \phi(t)\right\|_{1}^{2}+\int_{T}^{t}(1+\tau)^{\alpha+l}\left\|\partial_{x}^{l+1} \phi(\tau)\right\|^{2} d \tau \leq D_{l+1}(E, T) C_{l+1}\left(\phi_{0}, \delta\right)\right)(1+t)^{\alpha-\frac{1}{2}} \ln ^{12 l-6}(2+t)
$$

hold for $l=1, \ldots, k-1$, we now try to prove that (4.4) holds for $l=k$.

To this end, we need to deduce certain estimates on composite functions and to do so, we need the following result whose proof can be found in the proof of Theorem 4.3 of [5]

Lemma 4.3 ([5]) Let $F$ and $g$ be sufficiently smooth scalar functions, and $g$ is the function of $t, x$ and $g(t, \cdot) \in H^{l}(\mathbf{R}) \cap L^{\infty}(\mathbf{R})$ ( $l$ is a positive integer). Then $F(g)(t, \cdot)$ satisfies that

$$
\left\|\partial_{x}^{l} F(g(t, \cdot))\right\|_{L^{p}} \leq C\left(\|g(t)\|_{L^{\infty}}\right)\left\|\partial_{x}^{l} g(t, \cdot)\right\|_{L^{p^{\prime}}} \quad p \geq 1 .
$$

Based on Lemma 4.3, we first deduce an estimate on $\left\|\partial_{x}^{i}\left\{\left(\frac{f^{\prime \prime \prime}(w)}{f^{\prime \prime}(w)}\right) w_{x}^{2}\right\}(t)\right\|$

Lemma 4.4 For each $i \geq 1$, we have

$$
\left\|\partial_{x}^{i}\left\{\left(\frac{f^{\prime \prime \prime}(w)}{f^{\prime \prime}(w)}\right) w_{x}^{2}\right\}(t)\right\|^{2} \leq C \delta^{4}(1+t)^{-\left(i+\frac{5}{2}\right)} .
$$

Proof: For each $p \geq 1$, it is easy to see from Lemma 4.3 that for any smooth function $g(u)$

$$
\left\|\partial_{x}^{i} g(w)(t)\right\|_{L^{p}} \leq C\left\|\partial_{x}^{i} w(t)\right\|_{L^{p}} \leq C \delta(1+t)^{-\frac{1}{2}\left(i-\frac{1}{p}\right)}, \quad i \geq 1
$$

Moreover, Lemma 2.1 implies that

$$
\left\|\partial_{x}^{i}\left(w_{x}^{2}\right)(t)\right\|_{L^{p}} \leq C \delta(1+t)^{-\frac{1}{2}\left(i+3-\frac{1}{p}\right)}, \quad i \geq 1, \quad 1 \leq p \leq \infty .
$$

Having obtained (4.17) and (4.18), the proof of (4.16) is straightforward. This completes the proof of Lemma 4.4.

Now we turn to deduce the $L^{2}$-norm estimates on the following two terms

$$
\left\{\begin{aligned}
I_{1}= & \partial_{x}^{k}(f(w+\phi)-f(w))-\partial_{x}^{k} \phi \int_{0}^{1} f^{\prime}(\theta \phi+w) d \theta \\
I_{2}= & \partial_{x}^{k+2}\left(f(w+\phi)-f^{\prime}(\phi+w) \partial_{x}^{k+2}(\phi+w)\right. \\
& -(k+2) f^{\prime \prime}(\phi+w) \partial_{x}^{k+1}(\phi+w) \partial_{x}(\phi+w) .
\end{aligned}\right.
$$

For this purpose, we first have from (4.3), the induction assumption (4.14) and Sobolev's inequality that

$$
\begin{aligned}
&\|\phi(t)\|_{L^{\infty}}^{2} \leq C(E, T)\left(\left\|\phi_{0}(x)\right\|_{2}^{2}+\delta^{2}+\left\|\phi_{0}(x)\right\|_{L^{1}}^{2}\right)(1+t)^{-1} \ln ^{4}(2+t)\left(1+\left\|\phi_{0}(x)\right\|_{L^{1}}+\left\|\phi_{0}(x)\right\|_{2}\right)^{2} \\
&\left\|\partial_{x}^{j} \phi(t)\right\|_{L^{\infty}}^{2} \leq C(E, T)\left(\left\|\phi_{0}(x)\right\|_{j^{2} 2}^{2}+\delta^{2}+\left\|\phi_{0}(x)\right\|_{L^{1}}^{2}\right)(1+t)^{-j-1} \ln ^{12 j}(2+t) \\
& \times\left(1+\left\|\phi_{0}(x)\right\|_{L^{1}}+\left\|\phi_{0}(x)\right\|_{j+2}\right)^{12 j-2}, \quad 1 \leq j \leq k-2
\end{aligned}
$$




$$
\begin{aligned}
&\left\|\partial_{x} \phi(t)\right\|_{L^{\infty}}^{2} \leq C(E, T)\left(\left\|\phi_{0}(x)\right\|_{2}^{2}+\delta^{2}+\left\|\phi_{0}(x)\right\|_{L^{1}}^{2}\right)(1+t)^{-\frac{3}{2}} \ln ^{6}(2+t)\left(1+\left\|\phi_{0}(x)\right\|_{L^{1}}+\left\|\phi_{0}(x)\right\|_{2}\right)^{4}, \\
&\left\|\partial_{x}^{j} \phi(t)\right\|_{L^{\infty}}^{2} \leq C(E, T)\left(\left\|\phi_{0}(x)\right\|_{j+1}^{2}+\delta^{2}+\left\|\phi_{0}(x)\right\|_{L^{1}}^{2}\right)(1+t)^{-j} \ln ^{12(j-1)}(2+t) \\
& \times\left(1+\left\|\phi_{0}(x)\right\|_{L^{1}}+\left\|\phi_{0}(x)\right\|_{j+2}\right)^{12 j-14}, \quad 2 \leq j \leq k-1,
\end{aligned}
$$

and

$$
\begin{aligned}
\left\|\partial_{x}^{j} \phi(t)\right\|^{2} \leq & C(E, T)\left(\left\|\phi_{0}(x)\right\|_{j}^{2}+\delta^{2}+\left\|\phi_{0}(x)\right\|_{L^{1}}^{2}\right)(1+t)^{-\left(j-\frac{1}{2}\right)} \ln ^{12 j-18}(2+t) \\
& \times\left(1+\left\|\phi_{0}(x)\right\|_{L^{1}}+\left\|\phi_{0}(x)\right\|_{j}\right)^{12 j-20}, \quad 2 \leq j \leq k .
\end{aligned}
$$

It is worth to pointing out that although we can derive a better decay estimate on $I_{2}$, the estimates (4.22) - (4.24) are designed to deduce the coefficient $C_{k+1}(\varphi, \delta)$ on the right-hand side of (4.26) inductively.

From the induction assumption (4.14), (4.20)-(4.24), we can deduce the following estimates on $I_{1}$ and $I_{2}$

Lemma 4.5 Under the assumption (4.14), we have

$$
\int_{T}^{t}(1+\tau)^{\alpha+k}\left\|I_{1}(\tau)\right\|^{2} d \tau \leq C(E, T) C_{k+1}\left(\phi_{0}, \delta\right)(1+t)^{\alpha-\frac{1}{2}} \ln ^{12 k-6}(2+t)
$$

and

$$
\begin{aligned}
\int_{T}^{t}(1+\tau)^{\alpha+k}\left\|I_{2}(\tau)\right\|^{2} d \tau & \leq \frac{1}{16} \int_{T}^{t}(1+\tau)^{\alpha+k}\left\|\partial_{x}^{k+1} \phi(\tau)\right\|^{2} d \tau \\
& +C(E, T) C_{k+1}\left(\phi_{0}, \delta\right)(1+t)^{\alpha-\frac{1}{2}} \ln ^{12 k-6}(2+t) .
\end{aligned}
$$

Proof: We only prove (4.26) since the proof of (4.25) is less complicated and thus we omit the details for brevity.

(4.26) will be proved for the cases of $k=2$ and $k>2$, respectively. First, we show that (4.26) holds for the case of $k=2$. For such a case, we can deduce that

$$
\begin{aligned}
\int_{T}^{t}(1+\tau)^{\alpha+2}\left\|I_{2}(\tau)\right\|^{2} d \tau & \leq \underbrace{O(1) \int_{T}^{t} \int_{R}(1+\tau)^{\alpha+2}\left(\partial_{x}(w+\phi)\right)^{8}+\left(\partial_{x}(w+\phi)\right)^{4}\left(\partial_{x}^{2}(w+\phi)\right)^{2} d x d \tau}_{I_{2,1}} \\
& \underbrace{+O(1) \int_{T}^{t} \int_{R}(1+\tau)^{\alpha+2}\left(\partial_{x}^{2}(w+\phi)\right)^{4} d x d \tau .}_{I_{2,2}}
\end{aligned}
$$

From Lemma 2.1, (4.7), the induction assumption (4.14) and the Sobolev's inequality, we get

$$
I_{2,1} \leq C(E, T)\left(\left\|\phi_{0}(x)\right\|_{2}^{2}+\delta^{2}+\left\|\phi_{0}(x)\right\|_{L^{1}}^{2}\right)(1+t)^{\alpha-1} \ln ^{12}(2+t)\left(1+\left\|\phi_{0}(x)\right\|_{L^{1}}+\left\|\phi_{0}(x)\right\|_{2}\right)^{10}
$$


and

$$
\begin{aligned}
I_{2,2} & \leq O(1)\left(\int_{T}^{t}(1+\tau)^{\alpha+2}\left\|\partial_{x}^{2} \phi(\tau)\right\|^{2}\left\|\partial_{x}^{2} \phi(\tau)\right\|_{L^{\infty}}^{2} d \tau+\delta^{2}(1+t)^{\alpha-\frac{3}{2}}\right) \\
& \leq \frac{1}{16} \int_{T}^{t}(1+\tau)^{\alpha+2}\left\|\partial_{x}^{3} \phi(\tau)\right\|^{2} d \tau+O(1)\left(\int_{T}^{t}(1+\tau)^{\alpha+2}\left\|\partial_{x}^{2} \phi(\tau)\right\|^{6} d \tau+\delta^{2}(1+t)^{\alpha-\frac{3}{2}}\right) \\
& \leq \frac{1}{16} \int_{T}^{t}(1+\tau)^{\alpha+2}\left\|\partial_{x}^{3} \phi(\tau)\right\|^{2} d \tau+C(T) C_{3}\left(\phi_{0}, \delta\right)(1+t)^{\alpha-\frac{3}{2}} \ln ^{18}(2+t)
\end{aligned}
$$

Putting (4.27), (4.28), and (4.29) together yields (4.26) for $k=2$.

For the case $k>2$, we have

$$
\begin{aligned}
& \int_{T}^{t}(1+\tau)^{\alpha+k}\left\|I_{2}(\tau)\right\|^{2} d \tau \\
\leq & O(1) \sum_{2 \leq \rho \leq k+2} \int_{T}^{t} \int_{\mathbf{R}}(1+\tau)^{\alpha+k}\left|\left(\partial_{x}(w+\phi)\right)^{\alpha_{1}} \cdots\left(\partial_{x}^{k}(w+\phi)\right)^{\alpha_{k}}\right|^{2} d x d \tau \\
\leq & \underbrace{O(1) \int_{\mathbf{R}}^{t}(1+\tau)^{\alpha+k}\left|\partial_{x}(w+\phi)\right|^{2(k+2)} d x d \tau}_{T} \\
& +\underbrace{O(1)}_{2 \leq \rho \leq k+2} \int_{T}^{t} \int_{\mathbf{R}}(1+\tau)^{\alpha+k}\left|\left(\partial_{x}(w+\phi)\right)^{\alpha_{1}} \cdots\left(\partial_{x}^{k}(w+\phi)\right)^{\alpha_{k}}\right|^{2} d x d \tau,
\end{aligned}
$$

where $\alpha_{1}, \ldots, \alpha_{k}$, and $\rho$ satisfy

$$
\left\{\begin{array}{c}
\alpha_{1}+\alpha_{2}+\cdots+\alpha_{k}=\rho \\
\alpha_{1}+2 \alpha_{2}+\cdots+k \alpha_{k}=k+2
\end{array}\right.
$$

It is easy to see from Lemma 2.1, (4.3), (4.7), and (4.22) that

$$
I_{k, 1} \leq C(T)\left(\left\|\phi_{0}(x)\right\|_{2}^{2}+\delta^{2}+\left\|\phi_{0}(x)\right\|_{L^{1}}^{2}\right)(1+t)^{\alpha-\frac{1}{2}} \ln ^{4 k+2}(2+t)\left(1+\left\|\phi_{0}(x)\right\|_{L^{1}}+\left\|\phi_{0}(x)\right\|_{2}\right)^{4 k} .
$$

To estimate $I_{k, 2}$ in (4.30), we only deal with the case $\alpha_{k} \neq 0$ since the other cases are less complicated. In such a case the assumption $k>3$ and (4.31) implies that $\alpha_{k}=1$ and consequently we obtain from Lemma 2.1 and (4.22)-(4.24) that

$$
\begin{aligned}
I_{k, 2} \leq & O(1) \int_{T}^{t}(1+\tau)^{\alpha+k}\left(\prod_{j=1}^{k-1}\left\|\partial_{x}^{j}(\phi+w)(\tau)\right\|_{L^{\infty}}^{2 \alpha_{j}}\right)\left\|\partial_{x}^{\alpha_{k}}(\phi+w)(\tau)\right\| \\
\leq & C(T)(1+t)^{\alpha-\frac{1+\alpha_{1}}{2}}(\ln (2+t))^{12 k-6+12(2-\rho)+6 \alpha_{1}} \\
& \times\left(\left\|\phi_{0}(x)\right\|_{2}^{2}+\delta^{2}+\left\|\phi_{0}(x)\right\|_{L^{1}}^{2}\right)\left(1+\left\|\phi_{0}(x)\right\|_{L^{1}}+\left\|\phi_{0}(x)\right\|_{2}\right)^{12 k-8+12(2-\rho)+6 \alpha_{1}} \\
\leq & C(T) C_{k+1}\left(\phi_{0}, \delta\right)(1+t)^{\alpha-\frac{1}{2}} \ln ^{12 k-6}(2+t) .
\end{aligned}
$$


Here we have used the facts that $2 \leq \rho \leq 3$ and $\rho=3$ if and only if $\alpha_{1}=2$ which are direct consequence of (4.31) and the facts that $\alpha_{k}=1, k \geq 3$.

(4.32) together with (4.33) yields (4.26) for the case $k>2$ and this complete the proof of Lemma 4.5 .

With the above preparations in hand, we go on with the proof of (4.14) for $l=k$. To this end, we have by performing $(1+t)^{\alpha+k} \partial_{x}^{k} \phi \times \partial_{x}^{k}(2.12)+(1+t)^{\alpha+k} \partial_{x}^{k} \psi \times \partial_{x}^{k}(2.13)+$ $(1+t)^{\alpha+k} \partial_{x}^{k+1} \phi \times \partial_{x}^{k+1}(2.12)+(1+t)^{\alpha+k} \partial_{x}^{k+1} \psi \times \partial_{x}^{k+1}(2.13)(k \geq 2)$ and integrating the resulting identity with respect to $t$ and $x$ over $[T, t] \times \mathbf{R}$ that

$$
\begin{aligned}
& (1+t)^{\alpha+k}\left\|\partial_{x}^{k} \phi(t)\right\|_{1}^{2}+\int_{T}^{t}(1+\tau)^{\alpha+k}\left\|\partial_{x}^{k+1} \phi(\tau)\right\|^{2} d \tau \\
& \leq \underbrace{(1+T)^{\alpha+k}\left\|\partial_{x}^{k} \phi(T)\right\|_{1}^{2}}_{H_{5}}+\underbrace{(\alpha+k) \int_{T}^{t}(1+\tau)^{\alpha+k-1}\left\|\partial_{x}^{k} \phi(\tau)\right\|_{1}^{2} d \tau}_{H_{6}}+\underbrace{O(1) \int_{T}^{t}(1+\tau)^{\alpha+k}\left\|\partial_{x}^{k+2} w(\tau)\right\|^{2} d \tau}_{H_{7}} \\
& +\underbrace{\left|2 \int_{T}^{t} \int_{\mathbf{R}}(1+\tau)^{\alpha+k} \partial_{x}^{k+1} \phi\left(I_{1}+\partial_{x}^{k} \phi \int_{0}^{1} f^{\prime}(w+\theta \phi) d \theta\right) d x d \tau\right|}_{H_{8}} \\
& +2 \underbrace{\left|\int_{T}^{t} \int_{\mathrm{R}}(1+\tau)^{\alpha+k} \partial_{x}^{k+1} \phi\left(I_{2}+f^{\prime}(w+\phi) \partial_{x}^{k+2} \phi+(k+2) f^{\prime \prime}(w+\phi) \partial_{x}^{k+1}(w+\phi) \partial_{x}(w+\phi)\right) d x d \tau\right|}_{H_{9}} \\
& +\underbrace{\left|2 \int_{T}^{t} \int_{\mathbf{R}}(1+\tau)^{\alpha+k} \partial_{x}^{k+1} \phi \partial_{x}^{k-1}\left\{\frac{f^{\prime \prime \prime}(w)}{f^{\prime \prime}(w)} w_{x}^{2}\right\} d x d \tau\right|}_{H_{10}}+\underbrace{\left|2 \int_{T}^{t} \int_{\mathbf{R}}(1+\tau)^{\alpha+k} \partial_{x}^{k+1} \phi \partial_{x}^{k+1}\left\{\frac{f^{\prime \prime \prime}(w)}{f^{\prime \prime}(w)} w_{x}^{2}\right\} d x d \tau\right|}_{H_{11}} .
\end{aligned}
$$

And we are now devoted to controlling $H_{5}-H_{11}$. First, we have from Lemma 2.1 and (4.1) that

$$
H_{5}+H_{7} \leq C(E, T)\left(\left\|\phi_{0}(x)\right\|_{k+1}^{2}+\delta^{2}\right)+C(T) \delta^{2}(1+t)^{\alpha-\frac{1}{2}} .
$$

Second, (4.7) and the induction assumption (4.14) imply

$$
H_{6} \leq \frac{1}{16} \int_{T}^{t}(1+\tau)^{\alpha+k}\left\|\partial_{x}^{k+1} \phi(\tau)\right\|^{2} d \tau+C(E, T) C_{k}\left(\phi_{0}, \delta\right)(1+t)^{\alpha-\frac{1}{2}} \ln ^{12 l-8}(2+t) .
$$

For $H_{8}$, we get from Lemma 2.1 and (4.21) that

$$
\begin{aligned}
& 2\left|\int_{T}^{t} \int_{\mathbf{R}}(1+\tau)^{\alpha+k} \partial_{x}^{k+1} \phi \partial_{x}^{k} \phi \int_{0}^{1} f^{\prime}(w+\theta \phi) d \theta d x d \tau\right| \\
\leq & O(1) M \int_{T}^{t}(1+\tau)^{\alpha+k}\left\|\partial_{x}^{k} \phi(\tau)\right\|^{2}\left(\left\|\partial_{x} \phi(\tau)\right\|_{L^{\infty}}+\left\|\partial_{x} w(\tau)\right\|_{L^{\infty}}\right) d \tau \\
\leq & C(E, T) C_{k+1}\left(\phi_{0}, \delta\right)(1+t)^{\alpha-\frac{1}{2}} \ln ^{12 k-6}(2+t),
\end{aligned}
$$


and (4.25) implies that

$$
\begin{aligned}
2\left|\int_{T} \int_{\mathbf{R}}(1+\tau)^{\alpha+k} \partial_{x}^{k+1} \phi I_{1} d x d \tau\right| \leq & \frac{1}{16} \int_{T}^{t}(1+\tau)^{\alpha+k}\left\|\partial_{x}^{k+1} \phi(\tau)\right\|^{2} d \tau \\
& +C(E, T) C_{k+1}\left(\phi_{0}, \delta\right)(1+t)^{\alpha-\frac{1}{2}} \ln ^{12 k-6}(2+t) .
\end{aligned}
$$

Consequently, we can deduce that

$$
H_{8} \leq \frac{1}{16} \int_{T}^{t}(1+\tau)^{\alpha+k}\left\|\partial_{x}^{k+1} \phi(\tau)\right\|^{2} d \tau+C(E, T) C_{k+1}\left(\phi_{0}, \delta\right)(1+t)^{\alpha-\frac{1}{2}} \ln ^{12 k-6}(2+t)
$$

Thirdly, we get from (2.8), (4.6), (4.7), and (4.26) that

$$
\begin{aligned}
H_{9} \leq & \frac{1}{16} \int_{T}^{t}(1+\tau)^{\alpha+k}\left\|\partial_{x}^{k+1} \phi(\tau)\right\|^{2} d \tau+2 \int_{T}^{t}(1+\tau)^{\alpha+k}\left\|I_{2}(\tau)\right\|^{2} d \tau+4(k+2) M \\
& \left.\times \int_{\bar{\eta}} \int_{T}^{t}(1+\tau)^{\alpha+k}\left\|\partial_{x}^{k+1} \phi(\tau)\right\|^{2} d \tau+C_{\infty} \int_{T}^{t}(1+\tau)^{\alpha+k-1}\left\|\partial_{x}^{k+1} \phi(\tau)\right\|^{2} d \tau\right) \\
\leq & \frac{5}{16} \int_{T}^{t}(1+\tau)^{\alpha+k}\left\|\partial_{x}^{k+1} \phi(\tau)\right\|^{2} d \tau+C(E, T) C_{k+1}\left(\phi_{0} \delta\right)(1+t)^{\alpha-1} \ln ^{12 l-6}(2+t) .
\end{aligned}
$$

At last, we have from (4.16) that

$$
H_{10}+H_{11} \leq \frac{1}{8} \int_{T}^{t} \int_{\mathbf{R}}(1+\tau)^{\alpha+k}\left\|\partial_{x}^{k+1} \phi(\tau)\right\|^{2} d \tau+C(T) \delta^{4}(1+t)^{\alpha-\frac{1}{2}} .
$$

Inserting (4.35), (4.36), and (4.39)-(4.41) into (4.34), we can deduce immediately that (4.14) holds for $l=k$. Thus Theorem 1.2 follows from the principle of mathematical induction.

\section{Acknowledgements}

This study was supported by two grants from the National Natural Science Foundation of China under contracts 10871151 and 10925103, respectively.

\section{Authors' contributions}

QX got the main idea and drafted the manuscript. YL participated in the calculations and designed the study. drafted the manuscript. JK participated in the calculations and design of the manuscript. All authors read and approved the final manuscript.

\section{Competing interests}

This paper is the necessary preparation for our later paper which is about the multidimensional case about the model system of a radiating gas. Compared with former results, although we ask the $L^{2}(\mathbf{R})$-norm of the initial perturbation to be small but the $L^{2}(\mathbf{R})$-norm of the first- and second-order derivatives of the initial perturbation with respect to the spatial variable $x$ can be large and consequently, the $H^{2}(\mathbf{R})$-norm of the initial perturbation can be large.

Received: 13 September 2011 Accepted: 10 April 2012 Published: 10 April 2012

\section{References}

1. Kawashima, S, Tanaka, Y: Stability of rarefaction waves for a model system of a radiating gas. Kyushu J Math. 58, 211-250 (2004). doi:10.2206/kyushujm.58.211

2. Matsumura, A, Nishihara, K: Global stability of the rarefaction waves of a one-dimensional model system for compressible viscous gas. Commun Math Phys. 144, 325-335 (1992). doi:10.1007/BF02101095

3. Il'in, AM, Oleinik, OA: Asymptotic behavior of solutions of the Cauchy problem for certain quaslinear equations for large time (Russian). Mat Sbornik. 51, 191-216 (1960) 
4. Ito, K: Asymptotic decay toward the planar rarefaction waves for viscous conservation laws in several dimensions. Math Models Meth Appl Sci. 6, 315-338 (1996). doi:10.1142/S0218202596000109

5. Li, T, Chen, Y: Nonlinear Evolution Equations. Science Press, Beijing, China (1997) (in Chinese)

6. Duan, $\mathrm{R}, \mathrm{Ma}, \mathrm{X}, \mathrm{ZhaO}, \mathrm{H}-\mathrm{J}$ : A case study of global stability of strong rarefaction waves of hyperbolic conservation laws with artificial viscosity. J Diff Equ. 228(1):259-284 (2006). doi:10.1016/j.jde.2006.02.005

7. Duan, $\mathrm{R}$, Zhao, $\mathrm{H}-\mathrm{J}$ : Global stability of strong rarefaction waves for the generalized KdV- Burgers equation. Nonlinear Anal. 66, 1100-1117 (2007). doi:10.1016/j.na.2006.01.008

8. Duan, R, Liu, H-X, Zhao, H-J: Nonlinear stability of rarefaction waves for the compressible Navier-Stokes equations with large initial perturbation. Trans Am Math Soc. 361, 453-493 (2009)

9. Duan, R-J, Fellner, K, Zhu, C-J: Energy method for multi-dimensional balance laws with non-local dissipation. J de Math Pures et Appl. 93(6):572-598 (2010). doi:10.1016/j.matpur.2009.10.007

10. Gao, W-L, Ruan, L-Z, Zhu, C-J: Decay rates to the planar rarefaction waves for a model system of the radiating gas in $n$ dimentions. J Diff Equ. 244, 2614-2640 (2008). doi:10.1016/j.jde.2008.02.023

11. Gao, W-L, Zhu, C-J: Asymptotic decay toward the rarefaction waves for a model system of the radiating gas in two dimentions. Math Models Methods Appl Sci. 18, 511-541 (2008). doi:10.1142/S0218202508002760

12. Harabetian, E: Rarefactions and large time behavior for parabolic equations and monotone schemes. Commun Math Phys. 114, 527-536 (1988). doi:10.1007/BF01229452

13. Hattori, Y, Nishihara, $K$ : A note on the stability of the rarefaction wave of the Burgers equation. Japan J Indust Appl Math. 8, 85-96 (1991). doi:10.1007/BF03167186

14. Iguchi, T, Kawashima, S: On space-time decay properties of solutions to hyperbolic-elliptic coupled systems. Hiroshima Math J. 32, 229-308 (2002)

15. Kawashima, S, Nishibata, S: Shock waves for a model system of a radiating gas. SIAM J Math Anal. 30, 95-117 (1999)

16. Nishihara, K, Zhao, H-J, Zhao, Y-C: Global stability of strong rarefaction waves of the Jin-Xin relaxation model for the $p$ system. Commun Part Diff Equ. 29(9\&10):1607-1634 (2004)

17. Zhu, C-J: Asymptotic behavior of solutions for p-system with relaxation. J Diff Equ. 180, 273-306 (2002). doi:10.1006/ jdeq.2001.4063

doi:10.1186/1029-242X-2012-8

Cite this article as: Xiao et al: Asymptotic behavior of rarefaction waves for a model system of a radiating gas. Journal of Inequalities and Applications 2012 2012:81.

\section{Submit your manuscript to a SpringerOpen ${ }^{\odot}$} journal and benefit from:

- Convenient online submission

- Rigorous peer review

- Immediate publication on acceptance

- Open access: articles freely available online

- High visibility within the field

- Retaining the copyright to your article

Submit your next manuscript at $\gg$ springeropen.com 